\title{
Scarred Patterns in Surface Waves
}

\author{
A. Kudrolli, ${ }^{1,2, \dagger}$ Mathew C. Abraham, ${ }^{1}$ and J. P. Gollub ${ }^{1,3, *}$ \\ ${ }^{1}$ Department of Physics, Haverford College, Haverford, PA 19041 \\ ${ }^{2}$ Department of Physics, Clark University, Worcester, MA 01610 \\ ${ }^{3}$ Physics Department, University of Pennsylvania, Philadelphia, PA 19104
}

(November 6, 2018)

\begin{abstract}
Surface wave patterns are investigated experimentally in a system geometry that has become a paradigm of quantum chaos: the stadium billiard. Linear waves in bounded geometries for which classical ray trajectories are chaotic are known to give rise to scarred patterns. Here, we utilize parametrically forced surface waves (Faraday waves), which become progressively nonlinear beyond the wave instability threshold, to investigate the subtle interplay between boundaries and nonlinearity. Only a subset (three main types) of the computed linear modes of the stadium are observed in a systematic scan. These correspond to modes in which the wave amplitudes are strongly enhanced along paths corresponding to certain periodic ray orbits. Many other modes are found to be suppressed, in general agreement with a prediction by Agam and Altshuler based on boundary dissipation and the Lyapunov exponent of the associated orbit. Spatially asymmetric or disordered (but time-independent) patterns are also found even near onset. As the driving acceleration is increased, the time-independent scarred patterns persist, but in some cases transitions between modes are noted. The onset of spatiotemporal chaos at higher forcing amplitude often involves a nonperiodic oscillation between spatially ordered and disordered states. We characterize this phenomenon using the concept of pattern entropy. The rate of change of the patterns is found to be reduced as the state passes temporarily near the ordered configurations of lower entropy. We also report complex but highly symmetric (time-independent) patterns far above onset in the regime that is normally chaotic.
\end{abstract}

PACS number(s): 47.54.+r, 05.45.+b

\section{INTRODUCTION}

Parametrically forced surface waves arising as a result of the Faraday instability have provided an excellent opportunity to study nonlinear pattern formation. One of the special features of this system is that the system size relative to the basic correlation length can be varied so that both the large aspect ratio and small aspect ratio limits can be explored. At large aspect ratio, all of the classic ordered patterns have been found, including stripes, hexagons, and squares; additional exotic structures such as quasicrystalline and superlattice patterns have also been found, as well as secondary instabilities giving rise to spatiotemporal chaos. Extensive references can be found in 11 [3].

The case of small aspect ratio has also been studied in rectangular and circular containers. Typically the wave patterns found near onset are either normal modes of the container or symmetrical combinations of these modes 国. For example, in the circular case the normal modes are Bessel functions of the radius multiplied by sinusoidal functions of the azimuthal angle. The effects of container shape can be either a nuisance or a benefit depending on one's point of view. One example of the usefulness of considering container geometry is the study by Lane et al. [5] in which the conceptual differences between square symmetry and square geometry were elucidated. On the other hand, finite size effects impede efforts to utilize amplitude equations to describe the wave dynamics.

The influence of the container shape is also a fundamental issue in the field of quantum chaos. There is known to be a close correspondence between certain finite quantum systems (or analogous systems supporting classical waves) and their particle (or ray-optic) counterparts [6,7]. Of particular interest are non-integrable quantum systems with classical counterparts that are chaotic, such as the billiard formed from two semicircles separated by two straight edges. For almost all initial conditions, a particle launched inside such a billiard will exhibit sensitive dependence on initial conditions - the hallmark of chaos. Experimental, numerical and theoretical studies have shown that the statistical behavior of the wavefunctions of the quantum or wave version of this system is distinctly different from the behavior for "integrable" geometries such as a square or circle [8 10]. Most notably, regions of high amplitude in the wavefunctions - called scars - are found along some of the deleted word periodic orbits of the classical counterpart [11].

Effects of this type were explored to a limited extent using parametrically forced surface waves by Blümel et al. [12]. Their experiment utilized water as a working fluid and high frequency excitation. They reported observations of "scarlets", that are ridge like structures consistent with a random superposition of plane waves [13] (but are not 
located along periodic orbits.) On the other hand, no clear evidence for the simpler "scarred" wavefunctions was given. The possible effects of hydrodynamic nonlinearity on the utility of the ray optics approach has also not been discussed. Nonlinearity is in principle important, since even infinitesimally above the onset of instability, saturation of the wave amplitude is produced by nonlinear effects.

In this paper we first present observations of the spatial modes of Faraday waves in a finite non-integrable geometry, close to onset where nonlinearity is as weak as possible, and the waves might be usefully described by quasi-classical (ray optics) methods. A low viscosity fluid in a stadium shaped container is used for this purpose. Scarred patterns that resemble the computed eigenfunctions of the stadium geometry are clearly evident, but some of the linear eigenfunctions are apparently suppressed. The relative suppression of certain modes has been plausibly explained by Agam and Altshuler [14] in terms of higher dissipation rates for those modes near the boundaries in comparison with the modes that are observed.

We then consider the evolution of the wave patterns as the degree of nonlinearity is increased. Transitions between modes are found at some driving frequencies, along with a general increase in spatial complexity. The scars that are characteristic of the linear eigenmodes are often evident substantially above onset. Finally, we consider the development of spatiotemporal chaos (STC) in the stadium geometry. Though the onset of STC is strongly dependent on the excitation frequency, the boundaries continue to play a large role, leading for example to coherent oscillations between symmetric and asymmetric states, a phenomenon that we study using the concept of pattern entropy.

\section{THEORETICAL BACKGROUND}

A fluid layer with a free surface is subjected to an oscillatory vertical acceleration of amplitude $a$. The surface is flat until a critical acceleration $a_{c}$ is reached, at which point the surface becomes unstable and standing wave patterns are observed that oscillate at half the driving frequency. The threshold acceleration depends on the frequency and the viscosity of the fluid. It is convenient to define a dimensionless driving parameter $\epsilon=\left(a-a_{c}\right) / a_{c}$ that measures the departure from onset and hence the degree of nonlinearity. The patterns are time-independent for a range of positive $\epsilon$ but eventually a secondary instability gives rise to spatiotemporal chaos. In this section, we briefly discuss the linear inviscid theory, the effect of viscosity, and the role of nonlinearity, as they pertain to the present investigation.

The linear stability theory for Faraday waves was first developed by Benjamin and Ursell [15]. We summarize it here because the quantum/classical correspondence occurs for linear waves. They started from the Euler (inviscid) equation of motion and the continuity equation for an ideal fluid with a free surface in an oscillating gravitational field, and simplified the equations by retaining only the linear terms appropriate for small amplitude waves. The surface deformation $h(\mathbf{x}, t)$ as a function of spatial coordinate $\mathbf{x}$ and time $t$ may be written as a superposition of normal modes $\psi_{i}(\mathbf{x})$ with coefficients $A_{i}(t)$ :

$$
h(\mathbf{x}, t)=\sum_{i} A_{i}(t) \psi_{i}(\mathbf{x})
$$

where $\psi_{i}(\mathbf{x})$ is a complete orthogonal set of eigenfunctions of the Helmholtz equation,

$$
\left(\nabla^{2}+k_{i}^{2}\right) \psi_{i}(\mathbf{x})=0 .
$$

The sidewall boundary condition is imposed by setting the normal component of the velocity of the fluid at the wall to zero. This leads to a quantization condition on $k_{i}$ (the wavenumber). In addition, $k_{i}$ satisfies the dispersion relation which relates the frequency of oscillation $\omega$ of the fluid to the wavenumber:

$$
\omega^{2}=\tanh \left(k_{i} d\right)\left(\frac{\Gamma}{\rho} k_{i}^{3}+g k_{i}\right),
$$

where, $\rho$ is the fluid density, $\Gamma$ is the surface tension, $d$ is the mean fluid depth, and $g$ is the gravitational acceleration. In our experiment, the wave number is sufficiently large so that the surface tension term is much greater than the gravity term. The hyperbolic tangent factor is close to unity since $k_{i} d$ is large.

The time dependent amplitudes $A_{i}$ of these normal modes satisfy the Mathieu equation:

$$
\frac{d^{2} A_{i}}{d t^{2}}+k_{i} \tanh k_{i} h\left(k_{i}^{2} \frac{\Gamma}{\rho}+g-a \cos (\omega t)\right) A_{i}=0 .
$$

An instability occurs and the amplitude $A_{i}$ grows exponentially in time when the eigenvalue is in a band (known as the stability tongue) such that the frequency of oscillation of the fluid is half the driving frequency. The instability occurs at arbitrarily small driving amplitude in the absence of viscosity. 
Damping, which is provided by a number of distinct mechanisms in addition to bulk viscosity, can be included by means of a phenomenological linear damping term as reviewed in Ref. 16. Though treating damping in this way may not be fully adequate, the main effect is to reduce the width of the stability tongue in parameter space and raise the critical threshold to a finite amplitude. A proper theoretical treatment of instability in the viscous case has been given in Ref. [17], where the shapes of the computed stability boundaries were presented.

If the acceleration $a$ is slightly higher than $a_{c}$, all modes in a band $(\mathbf{k}-\Delta \mathbf{k} / 2, \mathbf{k}+\Delta \mathbf{k} / 2)$ are accessible and can be excited. The wavenumber width $\Delta k$ of the stability band for small $\epsilon$ has been estimated [18, 19] to be:

$$
\Delta k=8 \sqrt{2} \rho \nu \omega \sqrt{\epsilon} / 3 \Gamma,
$$

where $\nu$ is the kinematic viscosity of the fluid. For a suitable choice of $\epsilon, \rho$, and $\omega$, and assuming no interaction between modes, one then expects to find either single mode patterns or superpositions of a few modes whose thresholds lie in the window $(\mathbf{k}-\Delta \mathbf{k} / 2, \mathbf{k}+\Delta \mathbf{k} / 2)$.

The cumulative number of eigenvalues of the Helmholtz equation $N(k)$ is related to the geometry and is given by:

$$
N(k) \cong \frac{S}{4 \pi} k^{2} \mp \frac{L}{4 \pi} k,
$$

where $S$ is the area, $L$ is the perimeter of the stadium, and the negative or positive sign corresponds to Dirichlet or Neumann boundary condition respectively [6]. At high $k$, the perimeter term is negligible compared to the area term. Taking $\epsilon=0.01$ and using Eqs. 5, 6 with an area $S$ that is appropriate to the experiments reported here, one can estimate that the typical number of accessible modes is about 8 for a driving frequency of $70 \mathrm{~Hz}$.

What are the effects of nonlinearity? A nonlinear theory that describes regular Faraday wave patterns in large containers rather well has been given by Zhang and Viñals [20] and Chen and Viñals [21]. In this theory, an evolution equation is determined for the time derivative of the amplitude of a typical Fourier mode $B_{1}$ of the interfacial deformation. It may be expressed in the form

$$
\frac{d B_{1}}{d T}=\alpha B_{1}-g_{0} B_{1}^{3}-\sum_{m \neq 1} g\left(\theta_{m 1}\right) B_{m}^{2} B_{1}
$$

where $T$ is a slow time variable, the linear term is due to the basic instability, the cubic self-interaction term produces saturation of the wave pattern, and the coupling terms to other modes (which depend on their relative angle $\theta$ ) are also of cubic order. The constants have been computed, and the ratio $g(\theta) / g_{0}$ is of order unity and independent of $\epsilon$. This implies that coupling effects between the accessible modes may be substantial. The theory was able to explain the striking cascade of $2 \mathrm{n}$-fold patterns discovered by Binks and van de Water [2]. It also explains semiquantitatively the appearance of striped, square and hexagonal patterns observed in experiments using viscous fluids in large containers [1]. However, the amplitude equation is variational, and is only appropriate near onset. It cannot describe nonuniform patterns, secondary instabilities, or spatiotemporal chaos. An earlier approach that allowed spatially varying patterns was given by Milner [22].

The amplitude equations also ignore the effects of the boundary. For slightly viscous fluids in small containers, a large fraction of the dissipation occurs in the boundary layer and can in fact be the leading cause of dissipation [14, 23, 24]. In work stimulated by the experiments reported here, Agam and Altshuler [14] show that the dissipation near the boundary depends strongly on the nature of the mode.

\section{EXPERIMENTAL APPARATUS}

The apparatus is similar to that used by Gluckman et al. in Ref. [25]. Fig. 1 shows a schematic diagram of the experimental setup. The stadium shaped container made of Delrin has the following dimensions: depth $d=1.25 \mathrm{~cm}$, radius of semicircles $r=3 \mathrm{~cm}$, and length of straight edge $l=4.5 \mathrm{~cm}$. The top and bottom plates of the container are made of glass to allow the transmission of light. The fluid is silicone oil of kinematic viscosity $0.02 \mathrm{~cm}^{2} \mathrm{~s}^{-1}$, chosen for its stable surface tension and good wetting characteristics. To minimize meniscus waves, a brim full boundary condition was prepared by machining a ledge in the boundary at the same height as the fluid. Therefore the fluid meets the ledge at $90^{\circ}$. By maintaining the fluid under brim full conditions, the fluid surface is pinned to the ledge and boundary dissipation is reduced. This situation has been modeled as a Dirichlet boundary condition $(\psi=0)$ [26]. The container is rigidly attached to an electromagnetic shaker (Vibration Test Systems Model 40C) and the acceleration is measured with an accelerometer. The apparatus is placed within a temperature-controlled environment. The driving frequency is selected to be greater than $55 \mathrm{~Hz}$ to be in the capillary wave limit, but less than $75 \mathrm{~Hz}$ to prevent the density of modes from becoming too high. 
The patterns are imaged with shadowgraph techniques. The specific implementation is discussed in depth in Ref. 25. Light from an expanded and collimated incident beam is collected and imaged onto a CCD (charge-coupled device) video camera via a large collecting lens and the camera lens. The resulting images can be interpreted by considering which rays of light reach the CCD plane after passing through the fluid. The relatively small aperture of the camera lens restricts the rays that reach the CCD. All the rays that leave the fluid surface at an angle measured from the normal that is greater than a critical angle (typically about $10^{-2}$ radians) are blocked. Since the critical angle is so small, light is collected only from the nearly horizontal regions of the wave surface. Therefore, the bright regions in an image corresponds to local extrema or antinodes of the wave pattern. Images are averaged over one video frame, $1 / 30 \mathrm{~s}$, which is more than a full cycle of the standing waves. The imaging process is nonlinear in the wave height, but a quantitative model for the measured intensity was presented and tested in Ref. [25].

\section{PATTERNS NEAR ONSET}

We made a survey of the time-independent wave patterns near onset over the range 55 to $65 \mathrm{~Hz}$ by changing the frequency in $0.1 \mathrm{~Hz}$ steps. In order to obtain useful statistics for the surface wave patterns, a systematic procedure was followed: for each selected frequency, $a_{c}$ was first measured to within $0.1 \%$ and then $\epsilon$ was raised to 0.01 , the smallest value that could be maintained accurately. The threshold $a_{c}$ is $3.1 \mathrm{~m} \mathrm{~s}^{-2}$ at $f=60 \mathrm{~Hz}$ and increases weakly with frequency .

A sequence of images from the survey for driving frequency $f$ between $60.1 \mathrm{~Hz}$ and $62.8 \mathrm{~Hz}$, and with an approximate spacing of $0.4 \mathrm{~Hz}$ (i.e. every fourth image), is shown in Fig. 2. This spacing is comparable to the experimentally observed increment $(0.3 \mathrm{~Hz})$ typically required to obtain a distinctly different pattern in this frequency range; it is greater then the computed mean level spacing (about $0.1 \mathrm{~Hz}$ in this frequency range as estimated from Eqns. 5 and 6.) Most of the observed patterns show the reflection symmetries of the stadium. Regions of large amplitude are often located along lines that would form periodic orbits of the classical analog. Since these regions are similar to those found in other numerical and experimental investigations [11,27], we refer to the patterns containing such enhancements as scarred patterns.

We compare the observed patterns with numerically computed eigenstates of the Laplace operator for the stadium geometry, obtained for comparable mean wavenumber using an algorithm due to Heller [28]. Sample computed eigenstates (selected from a large number of distinct patterns) are shown in Fig. 3. We find that some of the computed states resemble observed patterns. On the other hand, a one-to-one correspondence for sequences of eigenstates was definitely not observed. Furthermore, certain computed eigenstates that occur frequently such as the "whispering gallery" mode (Fig. 3d) were not observed in the full experimental frequency range.

Interestingly, most of the observed symmetric patterns resemble one of three basic classes of eigenstates shown in Fig. 3(a-c). For instance, Figs. 2(a,d) resemble the bouncing ball eigenstate Fig. 3(a); Figs. 2(g,h) are close to the longitudinal eigenstate of Fig. 3(b); and Figs. 2(b,e) are a combination of the longitudinal and bowtie eigenstates of Figs. 3(b,c). It is noteworthy that among the observed patterns are states such as Fig. 2(c) that do not have the reflection symmetries of the stadium. We refer to these as disordered patterns. Table I summarizes the percentages of the onset patterns that were visually judged to approximate particular computed scarred eigenstates in the frequency range 55 to $65 \mathrm{~Hz}$ at $\epsilon=0.01$. (Visual comparison was used because automated pattern recognition, which we attempted, was not sufficiently reliable.)

Since the discovery of scars, there have been a number of theoretical attempts to obtain a quantitative measure for scarring [29 31] based on eigenstate overlap, Wigner function overlap, and inverse participation ratios for the amplitudes in the vicinity of the scars. To utilize such measures experimentally, the local wave amplitude is required with high accuracy. The shadowgraph technique used here is quantitative but nonlinear [25] and does not provide this information. Development of a quantitative experimental measure of scarring has proven to be difficult even for linear probes.

We use the concept of "pattern entropy" as a tool to classify the patterns. Egolf, Melnikov, and Bodenschatz 32] have applied this concept successfully to measure the complexity of patterns observed in Rayleigh-Bénard convection. The pattern entropy is calculated from the power spectrum of the pattern. If $P(\mathbf{k})$ is the normalized two dimensional power spectrum of the pattern at time t, then the pattern entropy $E(t)$ is defined as:

$$
E(t)=-\sum_{\mathbf{k}} P(\mathbf{k}) \ln (P(\mathbf{k})) .
$$

Here $E(t)$ measures the spectral complexity of a pattern. If the image consists of just one Fourier mode of amplitude unity, then $E=0$; otherwise $E>0$. To minimize the effects of experimental noise, we sum contributions only in a band of wavenumbers centered at the mean wavenumber of the pattern with a rage of $\pm 25 \%$. In Table II, the 
approximate entropy ranges for the various types of patterns observed in the range 55-65 Hz are given. Note that the patterns are not distinguishable solely by their entropy, since some of the ranges overlap. However, the pattern entropy can be useful in studies of time dependence farther above onset, as we show in Sec. VI.

\section{PATTERNS BEYOND ONSET}

Here we examine the evolution of the wave patterns farther from onset, where the interactions between different Fourier components of the waves become increasingly nonlinear and the approximation of Eq. (2) becomes inapplicable. The patterns were observed to be time-independent while changing adiabatically with $\epsilon$ for $\epsilon<0$.3. On the other hand, they become weakly time-dependent for $\epsilon \geq 0.3$ at most frequencies.

The evolution toward time-dependence with increasing $\epsilon$ depends on the excitation frequency. Three examples of this evolution are shown in Figs. 4-6. For some driving frequencies, the patterns remain reflection-symmetric as $\epsilon$ is increased, but exhibit transitions from one spatial mode to another prior to the onset of time dependence, as in Fig. 5. In these cases, the transition to spatial disorder (asymmetry) tends to coincide with the onset of spatiotemporal chaos (STC).

It is important to note that as $\epsilon$ increases, the width of the stability tongue grows (see Eq.(5)): for example at $f=74.1 \mathrm{~Hz}$ and $\epsilon=0.2$ the number of accessible modes of the container is approximately 35 . Therefore, the observed mode switching might be a combined effect of the increase in the number of accessible modes and an increase in the degree of nonlinearity that couples them. It is remarkable that the container boundary continues to influence the patterns even at $\epsilon=0.252$ (Fig. $5 \mathrm{~d}$ ), where nonlinearity clearly plays a major role.

The variability of the nonlinear development is evident from examining the examples in Figs. 4-6. In Fig. 4, there is a general increase in complexity with $\epsilon$, but the dominant mode does not change. In Fig. 6, the near onset pattern is nearly obliterated even at $\epsilon=0.025$ by the growing complexity, and the pattern is also distinctly asymmetric, while remaining time-independent.

In one instance, a complex but symmetric time-independent pattern was observed at an unusually high driving amplitude of $\epsilon=0.8$ at a frequency of $65.0 \mathrm{~Hz}$, in a regime where spatiotemporal chaos is usually fully developed. The image shown in Fig. 7 was averaged over 3000 images taken over a period of 5 minutes to test for time-dependence. The lack of blurring demonstrates its time-independence.

\section{ROLE OF ORDERED STATES IN THE REGIME OF SPATIOTEMPORAL CHAOS}

For driving amplitudes just beyond the frequency-dependent onset of spatiotemporal chaos, the time dependence of the pattern is often intermittent; the patterns appear to oscillate between states that are relatively ordered and states that are relatively disordered (see the images in Fig. 8 and a corresponding web-based movie [33]). The power spectra of these typical patterns are also shown in Fig. 8 and indicate the greater complexity of the disordered case, where the power is distributed more uniformly on the ring corresponding to the dominant wavenumber. The time dependence and complexity of the patterns are monitored using two quantitative measures: (i) the rate of change of the pattern $R(t)$, and (ii) the entropy $E(t)$ as defined in Eq. (8). The rate of change $R(t)$ was calculated by subtracting two consecutive images $\mathrm{I}(\mathbf{x}, t+\Delta t)$ and $\mathrm{I}(\mathbf{x}, t)$ separated by a time interval of $\Delta t=0.36$ seconds, and calculating $R(t)$ according to the following formula:

$$
R(t)=c \sum(I(\mathbf{x}, t+\Delta t)-I(\mathbf{x}, t))^{2}
$$

where, $\mathbf{x}$ is the position, and $c$ is a constant scaling factor.

In Fig. 9(a), a section of the resulting quantity $R(t)$ is shown as function of time for $f=71.9 \mathrm{~Hz}$ and $\epsilon=0.55$. The data has been smoothed by averaging over four adjacent points. At every pronounced minimum of this smoothed data we find that the corresponding pattern is symmetric and appears to have long range order. At all other times the pattern is asymmetric and disordered. A graph of the corresponding entropy $E(t)$ is shown in Fig. 9(b). Examples of the ordered $(\mathrm{X})$ and disordered $(\mathrm{Y})$ states are shown in Fig. 8, where the location in time is indicated by symbols $\mathrm{X}$ $(t=124 \mathrm{~s})$ and $\mathrm{Y}(t=136 \mathrm{~s})$ respectively in Fig. 9. At $\mathrm{X}$, where the pattern entropy is low, $R(t)$ is small, while at $\mathrm{Y}$, where the pattern entropy is high, $R(t)$ is large. This correlation between $R(t)$, and $E(t)$ holds true for most of the other strong peaks and valleys.

For higher $\epsilon(>0.8)$ the oscillations diminish in strength and uniform STC is observed. In this regime, following Gluckman et al. 25], we obtained the time averaged pattern after adding 3000 instantaneous images obtained over five minutes. An example of an instantaneous pattern is shown in Fig. 10(a). The resultant average pattern is shown 
in Fig. 10(b). The average reveals considerable ordered structure including remnants of the bouncing ball states. This phenomenon is seen at most frequencies and persists up to $\epsilon \sim 1.6$. Beyond this point, scars were visually absent and the average patterns are locally parallel to the boundary, as observed in circular or square patterns by Gluckman et al. [25].

\section{DISCUSSION}

In this paper we have discussed the parametrically forced wave patterns formed in a stadium-shaped container containing a low viscosity fluid, as a function of driving frequency and amplitude. The patterns near onset $(\epsilon=0.01)$ were compared to a simple model consisting of linearized equations that reduce to the Helmholtz equation with Dirichlet boundary conditions (see Section II). While a large proportion of observed patterns resemble the numerically computed eigenstates of the stadium, many of the computed eigenstates (for instance the whispering gallery modes) were not observed in a scan with sufficient frequency resolution to detect them. The observed patterns may be broadly classified into three categories: (a) bouncing ball patterns, (b) longitudinal patterns, and (c) bowtie patterns, which have high amplitudes near corresponding periodic orbits. In addition, a significant number of disordered patterns (lacking in symmetry but time-independent) were observed near onset. Furthermore, the observed mode spacing ( $\sim 0.3 \mathrm{~Hz})$ is somewhat greater than the mean eigenvalue separation implied by Eq. $(6)(\sim 0.1 \mathrm{~Hz})$. These observations imply that the simplest model is inadequate even close to onset.

Recently, Agam and Altshuler have offered an explanation for the selection of modes at onset [14] by considering the stability of the periodic orbits corresponding to the scars. They argue that a threshold for excitation of a particular scarred pattern is given by:

$$
h>\gamma_{b}+\gamma_{p}+\lambda / 2,
$$

where $h$ is proportional to the rate that energy is pumped into the system (i.e. the driving amplitude), $\gamma_{b}$ is the dissipation rate in the bulk of the fluid, $\gamma_{p}$ is the dissipation near the perimeter, and $\lambda$ is the Lyapunov exponent of the ray orbit that predominantly scars the pattern. The bulk dissipation $\gamma_{b}=\nu k^{2}$ is the same for all the patterns and corresponds to approximately $2 \mathrm{sec}^{-1}$ in the frequency window used in the experiments. Therefore the appearance of a scarred pattern depends on a combination of the two remaining factors which are orbit-dependent. Stability of a pattern is favored both by a small Lyapunov exponent of the associated scarring orbit, and by small perimeter dissipation $\gamma_{p}$.

In the limit of high wavenumber $k$ relevant to our experiments, Agam and Altshuler derive an expression for the damping rate of scars due to boundary effects:

$$
\gamma_{p}=\frac{\sqrt{\omega \nu / 2}}{L} \sum_{i} \frac{\left(1-\cos ^{2}\left(\phi_{i}\right)\right)}{\cos \left(\phi_{i}\right)}
$$

where $\omega$ is the angular frequency, $\nu$ is the viscosity, $L$ is the length of the periodic orbit, and the sum is over all the collision points of the orbit with the boundary, $\phi_{i}$ being the angle between the orbit and a line perpendicular to the boundary at the collision point. The parameter $\gamma_{p}$ and the Lyapunov exponent have been calculated for most of the shortest periodic orbits and a long ergodic orbit in Ref. 114. The perimeter dissipation $\gamma_{p}$, which can be either smaller or larger than $\gamma_{b}$, varies between $0.2 \mathrm{sec}^{-1}$ and $3.0 \mathrm{sec}^{-1}$. The extreme values correspond to patterns scarred by the horizontal orbit and the ergodic orbit respectively.

Most of the features observed in the experiments near onset appear to be captured by Eq. (10). The bouncing ball orbit is prominent because the Lyapunov exponent is zero in that case, and the longitudinal orbit occurs because of relatively low perimeter dissipation. The whispering gallery orbits and others with angles that come close to $\pi / 2$ have particularly large boundary dissipation and are suppressed. Eq. 10 implies that if one increases the dissipation on the boundary so that it dominates, the longitudinal orbit will be the last to survive. This is precisely what we observe in the experiments when the level of the fluid is lowered, a change that results in higher perimeter dissipation because of motion of the contact line.

The theory just cited [14] is also able to account for the observed tendency of one scarred pattern to suppress other nearby eigenmodes through nonlinear interactions, as well as the existence of some asymmetric patterns. At some driving frequencies the patterns are observed to switch modes as $\epsilon$ is increased (Fig. 5). This occurs especially when the bowtie state is observed at onset, an observation that may be related to the larger perimeter dissipation and Lyapunov exponent of the bowtie mode.

At higher driving amplitude, additional nonlinear effects occur as indicated by the growth in spatial complexity, and no adequate theoretical treatment exists. However, the onset patterns are often robust, persisting in the presence 
of increasing spatial complexity. The boundaries remain influential even beyond the onset of time dependence. At sufficiently high $\epsilon(\sim 0.2)$, the onset patterns are no longer visible, though they persist in the time-average.

Strong intermittency in the degree of order of the patterns is observed in the regime of spatiotemporal chaos. Furthermore, the rate of change of the pattern $R(t)$ just above the STC onset is strongly correlated with the order as characterized by the entropy $E(t)$. The more ordered patterns evolve more slowly in time, a striking observation that remains to be explained. This tendency for ordered patterns to be more stable may be related to the complex but highly symmetric and time-independent pattern observed at atypically large acceleration (Fig. 7).

\section{ACKNOWLEDGMENTS}

We thank Oded Agam and Boris Altshuler for many useful discussions, and E.J. Heller for software to determine eigenstates of the stadium. Bruce Boyes provided technical help. This work was supported by the National Science Foundation under Grant DMR-9704301. A. K. acknowledges a grant from the Alfred P. Sloan Foundation.

$\dagger$ Email: akudrolli@clarku.edu

* Email: jgollub@haverford.edu

[1] A. Kudrolli and J. P. Gollub, Physica D 97, 133 (1996).

[2] D. Binks and W. van de Water, Phys. Rev. Lett. 78, 4043 (1997).

[3] A. Kudrolli, B. Pier, and J. P. Gollub, Physica D 123, 99 (1998).

[4] S. Douady and S. Fauve, Europhys. Lett. 6, 221 (1988).

[5] J. D. Crawford, J. P. Gollub, and D. Lane, Nonlinearity 6, 119 (1993).

[6] M. C. Gutzwiller, Chaos in Classical and Quantum Mechanics (Springer-Verlag, New York, 1991).

[7] E. J. Heller and S. Tomsovic, Physics Today 46 Issue 7, 38 (1993).

[8] A. Kudrolli, V. Kidambi, and S. Sridhar, Phys. Rev. Lett. 75, 822 (1995).

[9] O. Bohigas, M. J. Giannoni, and C. Schmit, Phys. Rev. Lett. 52, 1 (1984).

[10] A. V. Andreev, O. Agam, B. D. Simons, and B. L. Altshuler, Phys. Rev. Lett. 76, 3947 (1996).

[11] E. J. Heller, Phys. Rev. Lett. 53, 1515 (1984).

[12] R. Blümel, I. H. Davidson, W. P. Reinhardt, H. Lin, and M. Sharnoff, Phys. Rev. A 45, 2641 (1992).

[13] P. O'Connor, J. Gehlen, and E. J. Heller, Phys. Rev. Lett. 58, 1296 (1987).

[14] O. Agam and B. L. Altshuler, preprint: cond-mat/0004190.

[15] T. B. Benjamin and F. Ursell, Proc. Roy. Soc. London, Ser. A 225, 505 (1954).

[16] M. C. Cross and P. C. Hohenberg, Rev. Mod. Phys. 65, 851 (1993).

[17] K. Kumar and L. S. Tuckerman, J. Fluid Mech. 279, 49 (1994).

[18] S. Fauve and W. S. Edwards, J. Fluid Mech. 278, 123 (1994).

[19] K. Kumar, Proc. Roy. Soc. of London, Series A 452, 1113 (1996).

[20] W. Zhang and J. Viñals, J. Fluid Mech 336, 301, (1997).

[21] P. Chen and J. Viñals, Phys. Rev. Lett. 79, 2670 (1997).

[22] S. T. Milner, J. Fluid Mech. 225, 81 (1991).

[23] C. C. Mei and L. F. Liu, J. Fluid Mech. 59, 239 (1973).

[24] C. Martel, J. A. Nicolas, and J. M. Vega, J. Fluid Mech. 360, 213 (1998).

[25] B. J. Gluckman, C. B. Arnold, and J. P. Gollub, Phys. Rev. E 51, 1128 (1995).

[26] S. Douady, J. Fluid Mech. 221, 383 (1990).

[27] S. Sridhar, Phys. Rev. Lett. 67, 785 (1991).

[28] E. J. Heller, private communications.

[29] E. B. Bogomolny, Physica D 31, 169 (1988).

[30] O. Agam and S. Fishman, Phys. Rev. Lett. 73, 806 (1994).

[31] L. Kaplan and E. J. Heller, Phys. Rev. E 59, 6609 (1999).

[32] D. A. Egolf, I. V. Melnikov, and E. Bodenschatz, Phys. Rev. Lett. 80, 3228 (1998).

[33] Movies of the aperiodic oscillations between ordered and disordered states can be found at the following internet site: http://physics.clarku.edu/ akudrolli/stadium.html

FIG. 1. The Faraday wave and shadowgraph imaging apparatus. A stadium shaped container is rigidly attached to an electromagnetic shaker, which provides a sinusoidal oscillation of amplitude $a$. 
FIG. 2. Shadowgraph images of patterns observed in a stadium shaped container filled with silicone oil as a function of driving frequency $\left(\epsilon=0.01 ; \nu=0.02 \mathrm{~cm}^{2} \mathrm{~s}^{-1}\right)$. The frequency interval between displayed images is comparable to that required for the pattern to change significantly. (a) $60.1 \mathrm{~Hz}$; (b) $60.4 \mathrm{~Hz}$; (c) $60.8 \mathrm{~Hz}$; (d) $61.2 \mathrm{~Hz}$; (e) $61.6 \mathrm{~Hz}$; (f) $62.1 \mathrm{~Hz}$; (g) $62.4 \mathrm{~Hz}$; (h) $62.8 \mathrm{~Hz}$. The patterns are strongly influenced by the shape of the container. Both symmetric scarred patterns and asymmetric patterns are found (see text).

FIG. 3. Selected eigenfunctions of the Helmholtz equation for a stadium geometry identical to that used in the experiments. Wavenumbers are chosen to be in the range explored experimentally. (a) Bouncing ball mode; (b) longitudinal mode; (c) bowtie mode; (d) whispering gallery mode. A large proportion of the experimental patterns are similar to (a-c), which are scarred by periodic orbits (shown by lines) of the corresponding ray system. However eigenstates such as the whispering gallery mode (d) are not observed in the experiments.

FIG. 4. Steady patterns as a function of driving amplitude $\epsilon$ illustrate the effect of increasing nonlinearity at $f=62.8 \mathrm{~Hz}$. (a) $\epsilon=0.01$; (b) $\epsilon=0.025$; (c) $\epsilon=0.125$; (d) $\epsilon=0.249$. The influence of the scarred eigenfunction persists even in the presence of strong nonlinearity. Spatiotemporal chaos develops at higher $\epsilon$, beyond the range shown here.

FIG. 5. Same as Fig. 4, except that $f=71.4 \mathrm{~Hz}$. (a) $\epsilon=0.010$; (b) $\epsilon=0.020$; (c) $\epsilon=0.078$; (d) $\epsilon=0.252$. In this case, the scars persist but the dominant mode switches. The "bowtie" enhancement is lost and a "bouncing ball" enhancement appears.

FIG. 6. Same as Fig. 4, except that $f=74.7 \mathrm{~Hz}$. (a) $\epsilon=0.010$; (b) $\epsilon=0.015$; (c) $\epsilon=0.025$; (d) $\epsilon=0.058$ for $f=74.7 \mathrm{~Hz}$. In this case, the pattern becomes disordered at rather low $\epsilon$, but remains time-independent.

FIG. 7. A stationary symmetric pattern that is quite complex, observed at $\epsilon=0.8(f=65 \mathrm{~Hz})$, a regime where spatiotemporal chaos usually predominates. This image is an average over $5 \mathrm{~min}$; its sharpness demonstrates its stationarity.

FIG. 8. The onset of spatiotemporal chaos involves an irregular oscillation between (a) relatively ordered and (b) disordered patterns $(\epsilon=0.55$ and $f=71.9 \mathrm{~Hz})$. The corresponding power spectra are shown in $(\mathrm{c})$ and (d) respectively. The greater spectral isotropy in (d) contributes to a higher pattern entropy.

FIG. 9. (a) The rate of change $R(t)$ of the pattern as a function of time, as computed by differences between successive images. (b) The pattern entropy $E(t)$ as a function of time $(\epsilon=0.55$, and $f=71.9 \mathrm{~Hz})$. Both functions oscillate in time and they are strongly correlated; both are smaller for ordered patterns (e.g. point X, shown in Fig. 8(a)) than for disordered patterns (e.g. point Y, shown in Fig. 8(b)). A movie corresponding to this figure is available at the internet address in Ref. $[33]$.

FIG. 10. (a) Instantaneous and (b) time-averaged patterns in the regime of spatiotemporal chaos $(\epsilon=1.2, f=65 \mathrm{~Hz})$. We find that scars usually persist (except at very high $\epsilon$ ) and that the dominant pattern found at most frequencies is the one shown.

\begin{tabular}{lc}
\hline Class of Pattern & Percentage of Occurrence \\
\hline \hline Bouncing ball & $27.2 \% \pm 1.6 \%$ \\
Longitudinal & $50.1 \% \pm 3.3 \%$ \\
Bowtie & $13.2 \% \pm 2.5 \%$ \\
Disordered & $13.2 \% \pm 2.5 \%$ \\
\hline
\end{tabular}

TABLE I. The percentages of patterns of various types observed in a sample of 128 patterns near onset $(\epsilon=0.01)$, for driving frequency between 55 and $65 \mathrm{~Hz}$. A typical example of a bouncing ball pattern is shown Fig. 2(a), a longitudinal pattern in Fig. 2(g), a bowtie pattern in Fig. 2(e), and a disordered pattern in Fig. 2(c). Patterns with several components such as Fig. 2(b), which contains both the longitudinal and bowtie modes, are counted in both categories. Therefore the sum of the percentages is slightly over $100 \%$. 


\begin{tabular}{lc}
\hline Class of pattern & Approximate entropy ranges \\
\hline \hline Bouncing ball & 3.0 to 4.2 \\
Longitudinal & 4.2 to 5.6 \\
Bowtie & 4.5 to 5.8 \\
Disordered & 5.8 to 6.4 \\
\hline
\end{tabular}

TABLE II. Pattern spectral entropy, which is a measure of complexity and is used in classifying the observed patterns. The patterns are obtained in the driving frequency range 55 to $65 \mathrm{~Hz}$, slightly above onset $(\epsilon=0.01)$. 
This figure "fig1.gif" is available in "gif" format from: http://arxiv.org/ps/nlin/0002045v2 
This figure "fig2.gif" is available in "gif" format from: http://arxiv.org/ps/nlin/0002045v2 
This figure "fig3.gif" is available in "gif" format from: http://arxiv.org/ps/nlin/0002045v2 
This figure "fig4.gif" is available in "gif" format from: http://arxiv.org/ps/nlin/0002045v2 
This figure "fig5.gif" is available in "gif" format from: http://arxiv.org/ps/nlin/0002045v2 
This figure "fig6.gif" is available in "gif" format from: http://arxiv.org/ps/nlin/0002045v2 
This figure "fig7.gif" is available in "gif" format from: http://arxiv.org/ps/nlin/0002045v2 
This figure "fig8.gif" is available in "gif" format from: http://arxiv.org/ps/nlin/0002045v2 
This figure "fig9.gif" is available in "gif" format from: http://arxiv.org/ps/nlin/0002045v2 
This figure "fig10.gif" is available in "gif" format from: http://arxiv.org/ps/nlin/0002045v2 